\title{
QUOTIENTS OF SOLUTIONS OF LINEAR ALGEBRAIC DIFFERENTIAL EQUATIONS
}

\author{
LEE A. RUBEL
}

(Communicated by Kenneth R. Meyer)

\begin{abstract}
An example is given of two entire functions $f, g$ that satisfy linear differential equations with polynomial coefficients, whose quotient $h=f / g$ is entire, yet $h$ satisfies no such differential equation.
\end{abstract}

On the same day, Alfred van der Poorten and Alexander Eremenko independently asked me the same question that stems from the theorem of Ritt that if $f$ and $g$ are two simple exponential polynomials whose quotient $h$ is entire, then $h$ must also be a simple exponential polynomial, which means that $h(x)=\sum_{k=1}^{n} a_{k} e^{\lambda_{k} x}$, where $a_{k}$ and $\lambda_{k}$ are complex constants. They asked me whether the result remains true if "simple exponential polynomial" is replaced throughout by "entire solution of a linear differential equation with polynomial coefficients." Our theorem shows that the answer is no, using a "cousin" of a function shown to me by Walter Hayman for another purpose.

Theorem. Let

$$
\begin{aligned}
& f(x)=e^{2 \pi i x^{2}}-1, \\
& g(x)=e^{2 \pi i x}-1 .
\end{aligned}
$$

Then $h(x)=f(x) / g(x)$ is entire, but does not satisfy any linear differential equation with polynomial coefficients, even though $f$ and $g$ do.

Remark. The equations that $f$ and $g$ satisfy can be chosen to be monic, a condition that Eremenko asked for, in addition. This is trivial for $g$. As for $f$, observe that

$$
\begin{aligned}
f^{\prime}(x) & =(4 \pi i x) e^{2 \pi i x^{2}}, \\
f^{\prime \prime}(x) & =\left(-8 \pi^{2} x^{2}+4 \pi i\right) e^{2 \pi i x^{2}}, \\
f^{\prime \prime \prime}(x) & =-\left(16 \pi^{3} i x^{3}+24 \pi^{2} x\right) e^{2 \pi i x^{2}} .
\end{aligned}
$$

Received by the editors April 27, 1990.

1980 Mathematics Subject Classification (1985 Revision). Primary 34A20, 34A30.

The research of the author was partially supported by a grant from the National Security Agency. 
Now it is evident that

$$
f^{\prime \prime \prime}(x)+a x f^{\prime \prime}(x)+b f^{\prime}(x)=0
$$

for suitable constants $a$ and $b$.

Proof of the theorem. First, $h$ is entire because the denominator vanishes exactly at the integers, and the numerator vanishes at each integer. The idea behind the rest of the proof is that if $h(x)$ were to satisfy a linear differential equation with polynomial coefficients, then so would

$$
\tilde{h}(x)=\left(e^{2 i e x^{2}}-1\right) /\left(e^{2 i e x}-1\right),
$$

which is obtained by replacing $\pi$ in $h$ by $e$. But $\tilde{h}(x)$ has infinitely many poles, since most of the zeros of the denominator are not cancelled by zeros of the numerator. But a solution of a linear differential equation with polynomial coefficients can have singularities in the finite complex plane only at the zeros of the leading coefficient.

Now for the details. Suppose

$$
p_{n}(x) h^{(n)}(x)+p_{n-1}(x) h^{(n-1)}(x)+\cdots+p_{0}(x) h(x)=0,
$$

say as formal power series, where the $p_{j}$ are polynomials. Let $\varphi$ be an automorphism of $\mathbb{C}$, with $\varphi(\pi)=e$, and $\varphi(i)=i$, so that $\varphi$ fixes $\mathbb{Q}(i)$, the Gaussian rationals. Note that the $h^{(j)}(x)$ are rational functions of $\pi, x, e^{2 \pi i x}$, $e^{2 \pi i x^{2}}$ with coefficients in $\mathbb{Q}(i)$. Extend the action of $\varphi$ to $\mathbb{C}((x))$, the field generated by the formal power series in $x$, via

$$
\varphi\left(\sum a_{n} x^{n}\right)=\sum \varphi\left(a_{n}\right) x^{n}
$$

It is easy to see that $\varphi(x)=x, \varphi(\pi)=e, \varphi\left(e^{2 \pi i x}\right)=e^{2 e i x}, \varphi\left(e^{2 \pi i x^{2}}\right)=e^{2 e i x^{2}}$, and that $\varphi$ commutes with differentiation. Therefore, applying $\varphi$ to (8), we would get

$$
\tilde{p}_{n}(x) \tilde{h}^{(n)}(x)+\tilde{p}_{n-1}(x) \tilde{h}^{(n-1)}(x)+\cdots+\tilde{p}_{0}(x) \tilde{h}(x)=0,
$$

where

$$
\tilde{p}_{k}(x)=\varphi\left(p_{k}(x)\right), \quad k=0,1, \ldots, n .
$$

As explained above, (9) is impossible. Hence (8) is impossible and the theorem is proved.

\section{REFERENCES}

1. C. Ward Henson, Lee A. Rubel, and Michael F. Singer, Algebraic properties of the ring of general exponentials, Complex Variables Theory Appl. 13 (1989), 1-20.

2. J. F. Ritt, Algebraic combinations of exponentials, Trans. Amer. Math. Soc. 31 (1929), 654-679.

Department of Mathematics, University of Illinois, 1409 West Green Street, Urbana, ILLINOIS 61801 